\title{
Immune-Related Gene Spatzle4 and Its Differential Immune Responses against Microbes in the Silkworm, Bombyx Mori
}

\author{
Ying Xu ${ }^{1}$, Ji Liu ${ }^{1}$, Fengpeng $\mathrm{Li}^{1}$, Xuefang Wang ${ }^{1}$, Xihai $\mathrm{Li}^{1}$, Zhongyuan Shen ${ }^{2}$, Jinmei Wu ${ }^{1,2, ~ * ~}$ \\ ${ }^{1}$ College of Biotechnology, Jiangsu University of Science and Technology, Zhenjiang City, P. R. China \\ ${ }^{2}$ The Sericultural Research Institute, Chinese Academy of Agricultural Sciences, Zhenjiang City, P. R. China
}

\section{Email address:}

jwuus@hotmail.com (Jinmei Wu)

\section{To cite this article:}

Ying Xu, Ji Liu, Fengpeng Li, Xuefang Wang, Xihai Li, Zhongyuan Shen, Jinmei Wu. Immune-Related Gene Spatzle4 and Its Differential Immune Responses against Microbes in the Silkworm, Bombyx Mori. American Journal of Clinical and Experimental Medicine. Vol. 3, No. 6, 2015, pp. 344-349. doi: 10.11648/j.ajcem.20150306.14

\begin{abstract}
Spatzle is a key factor in Toll signaling pathway against microbe invasion. The spatzle4 gene from silkworm (Bmspz4) was successfully cloned in this study, it is the second cloned and studied gene in the Spz family besides Bmspz1 in the silkworm and it was cloned for the first time in silkworm integument. The spatzle4 gene expression was analyzed in different tissues of the third day fifth instar larvae and the highest expression was detected in the head, and secondly in integument. Microbe infection showed that BmSpz4 participated in immune response. The transcriptional expression of BmSpz4 was induced differentially between Gram-negative bacteria and Gram-positive bacteria or fungi. This study showed that Bmspz4 gene plays an important role in the innate immunity of integument of silkworm, Bombyx mori.
\end{abstract}

Keywords: Spatzle, Immune Response, Silkworm, Immune-Related Gene, Microbe

\section{Introduction}

Lepidopteran insects provide important model systems for innate immunity of insects. Caterpillars are among the most serious agricultural pests, and understanding of their immune systems has potential practical significance[1]. The silkworm is a model organism in the study of Lepidopteran. It is also an economically important insect. Sericulture has a history of more than 5,000 years. Sericulture production is a traditional industry in China. However, silkworm diseases have caused a great loss in economy. Due to the needs of industrial development, immune defense mechanism of the silkworm is a long-term focus of attention. Studies on the silkworm immune-related genes establish a good basis in the immune response against pathogen. This not only supplies a theory for disease-resistant abilities of silkworm variety, but also has significance in the development of new environmental pesticides and a new pathway for pest control.

Insects have no acquired immunity, and to resist the invasion of external microbes, they can only rely on their own natural immunity [2]. Innate immune system consists of humoral and cellular immune components. Cellular immune responses including blood cells mediated reactions, such as nodule formation, phagocytosis, and embedding, besides, humoral immune responses include activation of antimicrobial peptides (AMP) synthesis, prophenoloxidase system and melanization [3-5]. In response to microbial infection, the host activating multiple signaling pathways to induce the expression of AMPs, in which the main signaling pathway has Toll, IMD and JAK / STAT pathway[6].

Toll signaling pathway is the most studied signaling pathway. The Toll pathway in Drosophila and the interleukin-1 receptor (IL-1R)-NF-kB pathway in mammals are homologous signal transduction pathways [7]. Spatzle factor was first discovered in Drosophila, there are mainly two aspects of function in Drosophila. Spatzle is a key signal transducer for embryonic development and immune responses [7, 8]. It is an active ligand resulted from easter-mediated proteolysis of pro-spatzle, binds to the toll receptor on the ventral side of the syncytial embryo to initiate an intracellular signaling pathway for ventralization $[9,10]$. In Drosophila adults, prospatzle protein was cleaved into an active form by Spatzle-Processing Enzyme (SPE) [11]. Taussig's study on the Toll family showed that different family members could identify different microorganisms and trigger different antimicrobial reactions. Toll- 2 is involved in the immune response challenged by bacteria, Toll-1 and Toll-5 can activate the expression of antifungal peptide 
genes[12]. Toll is activated by interaction with a cleaved form of the cytokine-like polypeptide Spatzle[13, 14]. Pro-Spz processing and toll pathway activation induce the synthesis of antimicrobial peptides.

There are mainly two kinds of pathogen signal recognition pathway in Drosophila: Toll and Imd signal transduction pathways. In the innate immune response, a group of germline-encoded pattern recognition receptors (PRR) can recognize and bind to the conserved pathogen-associated molecular pattern (PAMP) in bacteria and fungi when microbes invade [15]. The Toll signal pathway is only activated by gram-positive bacteria and fungi. When gram-positive bacteria invade Drosophila, peptidoglycan recognition protein (PGRP) PGRP-SA as recognition receptors identifies cell wall lysine type peptidoglycan of the gram-positive bacteria, whereas after fungal infections, Gram-negative bacteria-binding protein (GNBP) GNBP-3 family can identify $\beta-1,3$ - glucan in fungal cell wall[16-18]. The Imd signal pathway can be activated by gram-negative bacteria and certain gram-positive bacteria [6, 19-21]. When gram-negative bacteria invade Drosophila, peptidoglycan recognition protein (PGRP) PGRP-LC as recognition receptors identifies cell wall DAP-type peptidoglycan of the gram-negative bacteria $[22,23]$. Spatzle protein is very similar to the nerve growth factor (NGF) of the higher animals as Spz has C-terminal peptide model with the similar arrangement of the disulfide bonds of the peptide fragments in NGF[24, 25].

Genome analysis found that silkworm substantially retain the innate immune signaling pathways, with a complete Toll and Imd signaling pathways. Spatzle is also a key factor in Toll signaling pathway in the silkworm, Bombyx mori [26]. It is known that there are 6 members in Spz family genes. Bmspzl was cloned and could activate the Toll signaling pathway as the Toll receptor ligand [27, 28].

In this study, a new immune-related gene BmSpz4 was cloned from the epidermis of silkworm, Bombyx mori. The evolutionary relationship of homologous genes of different species was analyzed. The microbe-induced expression profile of BmSpz4 and its function was also studied and possible molecular mechanism of silkworm immune response was explored.

\section{Materials and Methods}

\subsection{Material and Main Reagents}

Silkworm variety used is $\mathrm{Su} \cdot \mathrm{Jv} \times \mathrm{Ming} \cdot \mathrm{Hu}$. Plasmid carrier pMD18-T, RNAiso ${ }^{\mathrm{TM}}$ Plus for RNA extraction, M-MLV antiretroviral, LA Taq enzyme and PCR products plastic recycling kit, 3 'the Race kit were purchased from TaKaRa company and Escherichia coli (E.coli) DH10B was offered by our laboratory. All kinds of restriction enzymes were bought from Promega Biotechnology Company, plasmid kit was bought from Axygene Biotechnology Company, and primers were synthesized by Shanghai ShengGong biological technology company.

\subsection{Cloning of BmSpz4 Gene}

A DNA fragment of about $600 \mathrm{bp}$ was cloned and sequenced when we were trying to clone ATP-binding cassette genes from silkworm, Bombyx mori. By Blast search using the silkDB database, we found it is part of predicted spatzle-like gene in silkworm. To clone the complete ORF of the gene, RNA was extracted from epidermal tissue of fifth instar larvae Bombyx mori, thencDNA was synthesized by reverse transcription with RNA as a template. The complete open reading frame was cloned and sequenced. We also cloned and sequenced its 3 'end untranslated region using 3'-RACE technology.

Primers Spz4-F (5'-AGGATGCCGCAGGTTGCTC-3') and Spz4-R

ACATGTCCGCTTATGATGATGAGGATTTCCTTTGA-3') were used for BmSpz4 cDNA amplification by RT-PCR.

The thermal cycling conditions were 30 cycles of $94^{\circ} \mathrm{C}, 30$ $\mathrm{s} ; 60^{\circ} \mathrm{C}, 30 \mathrm{~s}$; and $72^{\circ} \mathrm{C}, 60 \mathrm{~s}$, followed by $10 \mathrm{~min}$ incubation at $72^{\circ} \mathrm{C}$.

The reaction product was cloned into pMD18-T vector (TakaRa) and confirmed by DNA sequence analysis.

\subsection{Sequence Analysis of BmSpz4}

The Spz4 ORF sequence was made BLASTN in silkworm genome database (http://silkworm.genomics.org.cn) to get the genome and mRNA sequence. For searching the DNA and protein databases [SWISS-PROT], the FASTA and BLAST programs (BLASTX, BLASTN and BLASTP) were used. Conserved motifs in protein and peptides were identified with the program PROSITE. The Open reading frame (ORF) was calculated by the program FRAMES. Exons and introns analysis were made by Sim4 (http://pbil.univ-lyon1.fr/sim4.php). The physical and chemical properties of protein and hydrophobic analysis were got through ProtParam and ProtScale. The $\mathrm{G}+\mathrm{C}$ content was calculated by the program WINDOW. Codon usage was performed with the program CODONFREQUENCY. The programs SMART and TMHMM were used for the prediction of transmembrane domains and the membrane topology of proteins. Multiple sequence alignment and construction of the phylogenetic tree was obtained by ClustalX (Version 1.83) and MEGA 4.0 softwares.

\subsection{Tissue Expression of $\mathrm{BmSpz} 4$}

To examine mRNA levels of BmSpz4 in different tissues, total RNA samples were isolated from dissected fat body, midgut, silk gland, head, integument, ovary, and testis of the fifth instar silkworm larvae as previously described. Semi-quantitative RT-PCR was conducted to measure spatzle4 gene expression in different tissues of the third day fifth instar larvae.

Primers sq-F ( $5^{\prime}$-AGGATGCCGCAGGTTGCTC-3' $)$ and sq-R ( 5'- CGATATTTCAAACGCCACGTCCGC -3') were used for BmSpz4 cDNA amplification by RT-PCR. Primers specific for silkworm eTIF4A (Accession Number: DQ443290.1) 
(eTIF4A-F:5'-CTGCAATCCAGCAACGCGCA-3', eTIF4A-R:5'- $\quad$ GCCAATCCACCTTGCGACGG-3')were used in the control reactions.

The cDNA fragments were amplified for 25 cycles at $94^{\circ} \mathrm{C}$ for $40 \mathrm{~s}, 60^{\circ} \mathrm{C}$ for $30 \mathrm{~s}$, and $72^{\circ} \mathrm{C}$ for $60 \mathrm{~s}$, followed by a final extension at $72^{\circ} \mathrm{C}$ for $10 \mathrm{~min}$. The PCR products were separated by gel electrophoresis and stained with ethidium bromide (EB).

\subsection{Induced BmSpz4 Expression in Integument after Microbe Injection}

To test the hypothesis that BmSpz4 participates in immune responses, the levelsof BmSpz4 mRNA was compared in integument after the silkworm larvae were injected with water and differentmicroorganisms.

Silkwormwas 5-4A,provided by Dr. Gui of Sericultural Research Institute, CAAS. Day 3,fifth instar silkworm larvae were injected with $\mathrm{H}_{2} \mathrm{O}(10 \mu \mathrm{l})$, Bacillus subtilis $\left(1.7 \times 10^{9}\right.$ cells $/ \mathrm{ml}, 10 \mu \mathrm{l})$, Escherichia coli $\left(6.4 \times 10^{9} \mathrm{cells} / \mathrm{ml}, 10 \mu \mathrm{l}\right)$, Saccharomyces cerevisiae $\left(1.6 \times 10^{9}\right.$ cells $\left./ \mathrm{ml}, 10 \mu \mathrm{l}\right)$. Above bacteria had been formalin-treated $\left(6 \%\right.$ formalin $25^{\circ} \mathrm{C}$ for 30 $\mathrm{min}$ ). Integument tissues were dissected at $24 \mathrm{~h}$ after injection for total RNA isolation. Then semi-quantitative RT-PCR was made as mentioned above.

\section{Results}

\subsection{Cloning and Sequencing of BmSpz Gene}

The complete open reading frame of BmSpz4 (Accession number: JN628282) was cloned and sequenced, and its 3 'end untranslated region was also cloned and sequenced using 3'-RACE technology. After sequencing, it was found that the actual ORF is the same with the corresponding predicted sequence in the SilkDB database. However, 3' untranslated region is not consistent with the prediction.

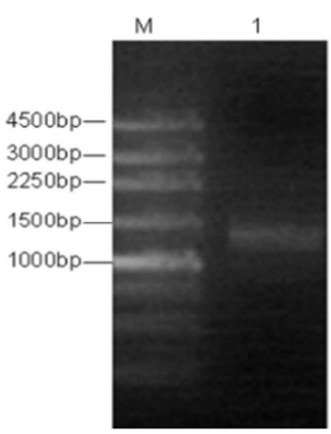

A

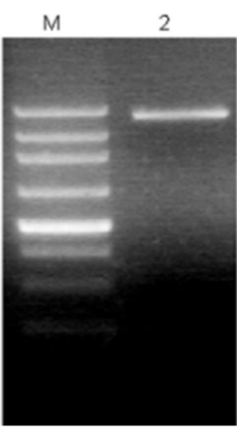

B

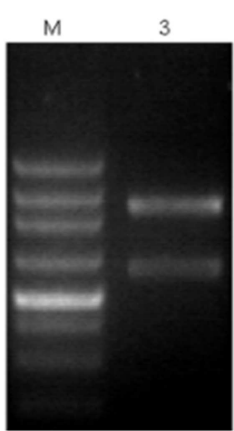

C
Fig. 1. PCR amplification of Spz4 gene (panelA) and identification of recombinant plasmid using restriction enzymatic digestion(panel $B \& C$ ).

M:250bp Ladder marker 1.PCR amplification product 2.Recombinant plasmid DNA containing foreign fragment digested with PstI 3.Recombinant plasmid DNA containing foreign fragment digested withXbaI and SalII.

The product was detected by $1.0 \%$ agarose gel electrophoresis and the expected band appeared between 1 $\mathrm{KB}$ and 1.5 KB marker bands (Figure 1-A). After target DNA extraction using gel recovery kit, the DNA fragment was inserted into the pMD18-T vector. Then we got positive clones through the blue-white selection. Shown in Figure1 are the digestion patterns of the recombinant plasmid digested with Pst I enzyme (panel B) and with $\mathrm{XbaI} / \mathrm{Sal}$ II (panel C).

\subsection{Structure and Molecular Evolution Analysis of BmSpz4 Gene}

The BmSpz4 is located in the chromosome 3 scaffold2927, only one copy is found in the genome. The gene has four exons and three introns, encoding 425 amino acid residues. The nucleotide sequence and the predicted BGIBMGA008841-TA gene sequence in SilkDB database are almost the same except one base (Fig2). ProtParam predict thatBmSpz4 molecular mass is $49.36 \mathrm{kD}$, isoelectric point5.31. It has no predicted N-terminal signal peptide. $\mathrm{BmSPZ}$ hydrophobicity is maximum 1.733 , minimum value is -3.011. The hydrophobic and hydrophilic regions of the protein amino acid sequence are staggered. SPZ has no transmembrane domains.

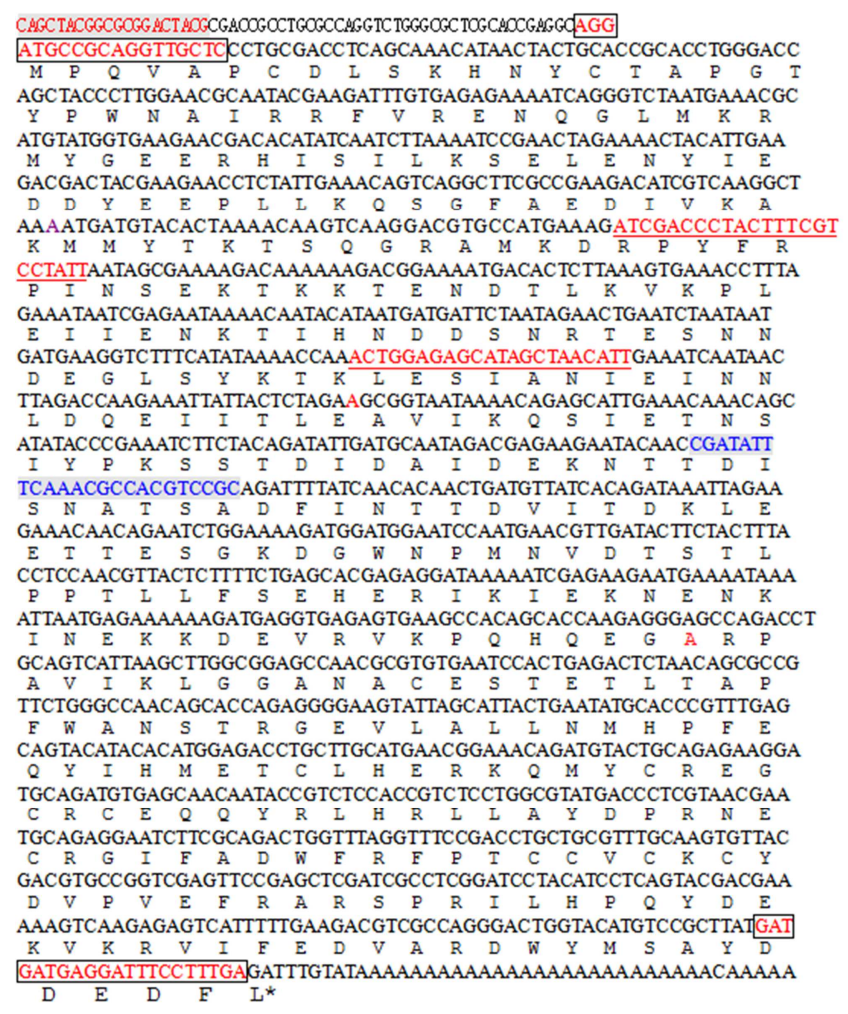

Fig. 2. The cDNA sequence and protein sequence of Spz4 gene in silkworm.

The primer sequences used for polymerase chain reaction of complete ORF of BmSpz4 are in the box. The primer of 3'-Race is underlined and highlighted. Shaded primers were used for semi-quantitative RT-PCR of BmSpz4 expression. The asterisk indicates the stop code.

Through the NCBI Blastp (http://blast.ncbi.nlm.nih.gov/Blast.cgi) procedures online for protein sequence alignment, the amino acid sequence of BmSPZ4 and those of Drosophila melanogaster, Nasonia vitripennis, Gambia mosquitoes, Anopheles gambiae, 
Tribolium castaneum, Acyrthosiphon pisum SPZs have closer relationship than those of mammalian SPZs. By sequence alignment, it is shown that the Spatzles from different insects have a highly conserved C-terminal cysteine-rich domain (Cys-knot domain) (Figure 3).

According to the amino acids sequence of different Spatzle proteins, the phylogenetic tree of Bmspz4 was constructed with homologous proteins from other species, including
Drosophila melanogaster, Nasonia vitripennis, Anopheles gambiae, Tribolium castaneum, Acyrthosiphon pisum, Apis mellifera, Harpegnathos saltator, Danio rerio, Mus musculus, Homo sapiens and Xenopus tropicalis. The result showed that the amino acid sequence of BmSPZ and those of insect SPZs have closer relationship than those of mammalian SPZs (Figure 4).

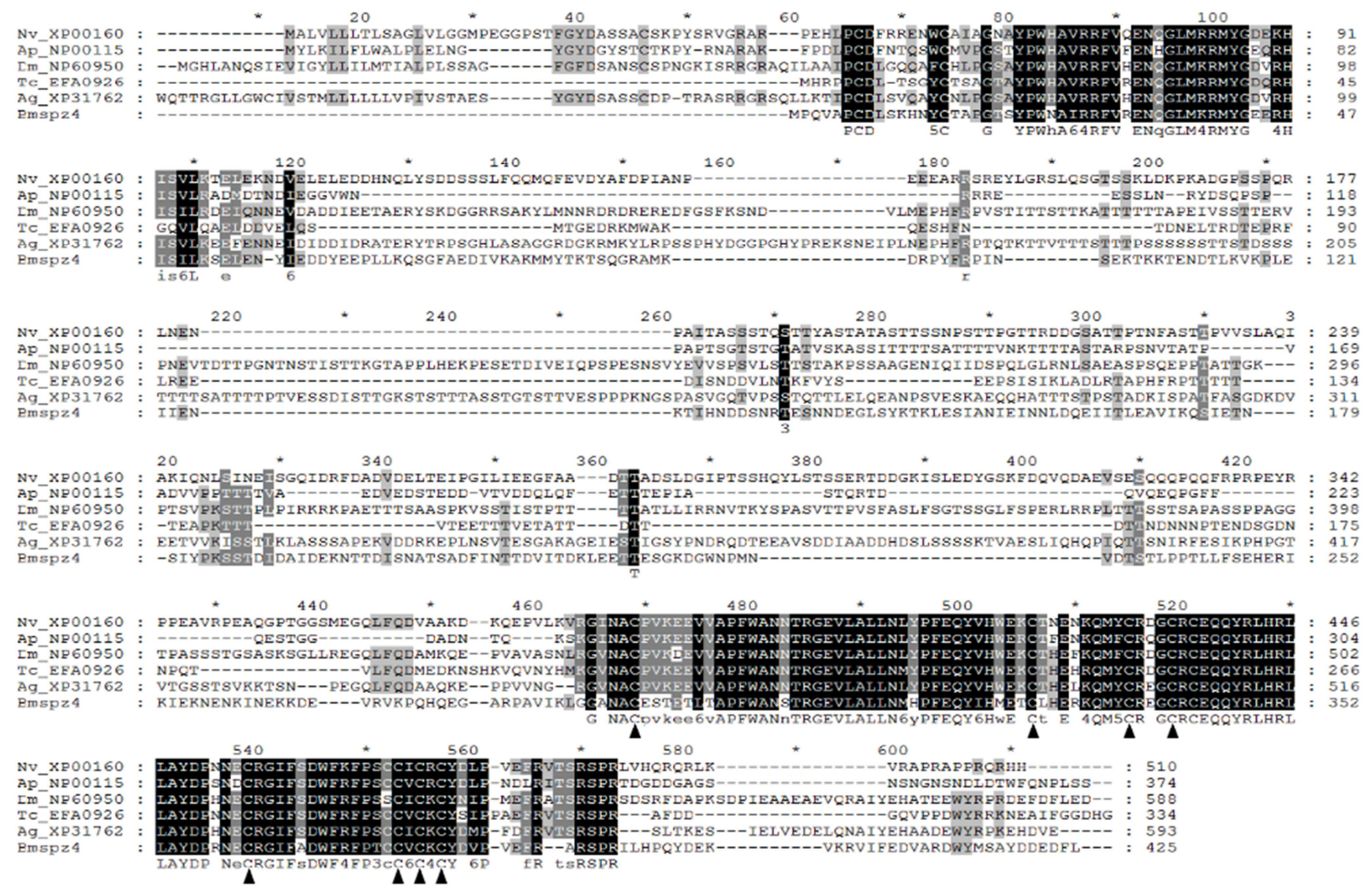

Fig. 3. Comparison of amino acids sequences of SPZ homologous proteins in silkworm and the other insects.

N. vitripennis (XP_001605307.1); A. pisum (NP_001153592.1); D. melanogaster (NP_609504.1); T. castaneum (EFA09263.1);

A. gambiae (XP 317626.3) and B. mori spz4 (AEP17031.1).

Solid triangles indicate the position of the conserved cysteines in the C-terminal Cys-knot domain

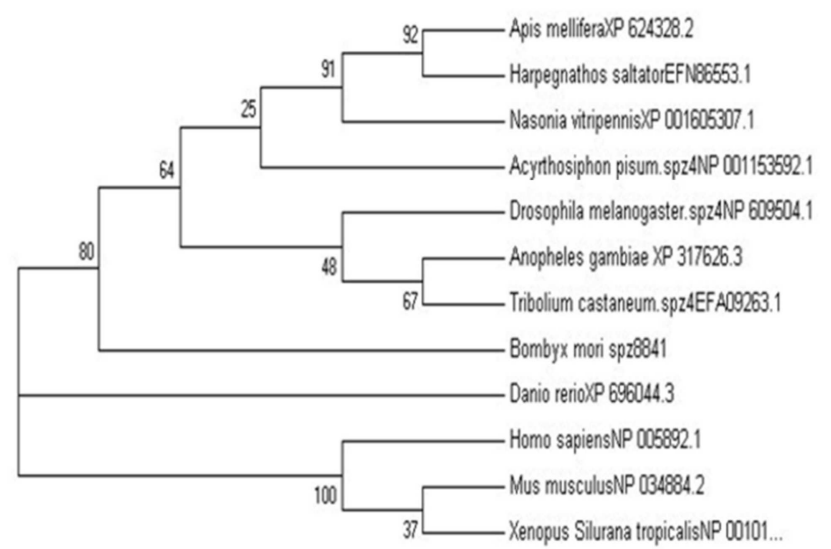

Fig. 4. Phylogenetic tree of Bmspz4 with its homologous proteins from other species.

\subsection{Tissue Expression Profile of BmSpz4}

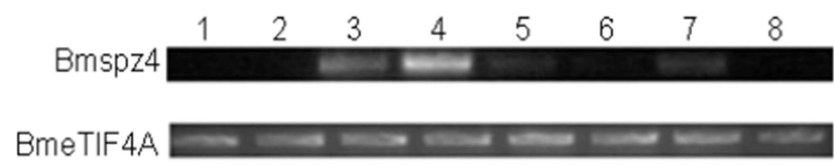

Fig. 5. Transcription profile of Spz4 gene in Bombyx mori various tissues on day 3 of the 5th instar.

The silkworm eTIF4A was used as an internal standard to normalize the templates.

1. Fat body 2.Midgut 3.Epidermis 4. Head 5. Malpighian tubule

6. Ovary 7.Testis 8.Anterior and Middle Silk gland

The result shows that BmSpz4 is expressed differently in tissues and is detected with the highest expression level in head, followed by a small amount of expression in the 
epidermis and testis. And there is no expression of this gene in the rest of the tissues (Figure 5).

\subsection{Microbe-Induced Expression Analysis of Bmspz4}

There was a significant increase at $24 \mathrm{~h}$ after the immune challenge with Bacillus subtilis and Saccharomyces cerevisia, and there was no obvious increase in the gene's transcription when the silkworm larvae were injected with Escherichia coli (Figure 6).

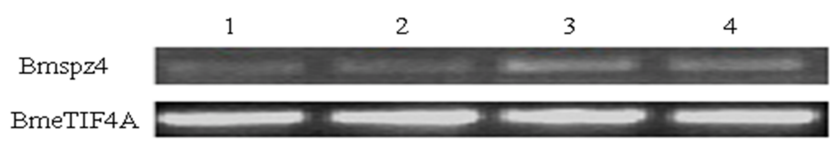

Fig. 6. Immune responsiveness of day 3, 5th instar silkworm larvae to different microbial challenge

The 3rd day fifth instar silkworm larvae were injected with water, E. coli, M. luteus, or S. cerevisiae cells (10ul each). Integument was collected from the injected larvae $24 \mathrm{hrs}$ later for total RNA extraction. RT-PCR and gel electrophoresis were performed. The silkworm eTIF4A was used as an internal standard to normalize the templates.

1. ddH2O injection

2. Escherichia coli injection

3. Bacillus subtilis injection, 4.Saccharomyces cerevisiae injection

\section{Discussion}

The immune-related gene spatzle4 of silkworm, Bombyx mori, was successfully cloned with bioinformatics and molecular biology methods. Bmspz4 is the second cloned and studied in BmSpz family besides Bmspzl and it was cloned for the first time in the skin of the silkworm.

The gene has four exons and three introns, encoding 425 amino acid residues. Its molecular mass is $49.36 \mathrm{kD}$, isoelectric point 5.31. It has no predicted N-terminal signal peptide. BmSPZ4 hydrophobicity is maximum 1.733, minimum value is -3.011 . The hydrophobic and hydrophilic regions of the protein amino acid sequence are staggered. SPZ has no transmembrane domains. The amino acid sequence of BmSPZ and those of Drosophila melanogaster, Nasonia vitripennis, Gambia mosquitoes, Anopheles gambiae, Tribolium castaneum, Acyrthosiphon pisumSPZ have closer relationship than those of mammalian SPZs. By sequence alignment and evolution analysis, it is shown that the newly cloned spatzle gene of silkworm is most closely related to the spatzle4 gene in Drosophila melanogaster, so it is named as BmSpz4.

By Semi-quantitative RT-PCR analysis, spatzle4 gene expression was analyzed in different tissues on the third day of the fifth instar larvae. The BmSpz4is detected the highest expression level in head, followed by integument and testis. And there is no expression of this gene in the rest of the tissues, which is different from Bmspz1 [28].

To test the hypothesis that BmSpz4 participates in immune responses, the levels of $\mathrm{BmSpz} 4 \mathrm{mRNA}$ was compared in integument after the silkworm larvae were injected with water and different microorganisms. There was a significant increase at $24 \mathrm{~h}$ after the immune challenge of Bacillus subtilis and Saccharomyces cerevisia, and there was no obvious increase in the gene's transcription when injected with Escherichia coli. As we all know that Bacillus subtilis and Saccharomyces cerevisia belong to Gram-positive bacteria and fungi respectively, and Escherichia coli belong to Gram-negative bacteria. Our results are consistent with those obtained using Drosophila melanogaster as an organism in microbe-induced response experiments [2].

Besides, present studies show that Toll pathway does not exist in the skin and only in the fat body of Drosophila[29, 30], but the Imd pathway does[29][31]. However, Spz4was cloned from the skin of the silkworm, which means the Toll pathway may exist in the skin of the silkworm. It may reveal possible new ways of the skin immunity of silkworm and other insects.

Our study found that 3'-untranslated region of BmSpz4 gene in our study does not match well with the corresponding 3 '-untranslated region of the $\mathrm{BmSpz} 4$ in the silkworm database SilkDB. The difference in sequence may be related to the silkworm varieties, which provides us new clues to study the relationship between structure and function of the immune-related genes.

Spatzle protein is very similar to the nerve growth factor (NGF) of the higher animals. By the preliminary analysis of the protein structure, BmSpz4 was found to have multiple Cys-knot structural features, which provides new clues for further study of the structure and function of $\mathrm{BmSpz} 4$ in the future.

The study of the new BmSpz4's function was still under the way, the specific mechanism is yet to be clarified.

\section{Conclusion}

In this study, The ORF and 3' end non-coding region of Bmspz4was cloned from Bombyx mori integument, which is the second cloned and studied gene in Spz family besides Bmspz1. Sequence alignment and evolution analysis showed that Bmspz4 gene is most closely related to Drosophila spz4. RT-PCR showed it has the highest expression in the head and followed by integument and testis. Microbial-induced expression showed that fungi and gram-positive bacteria invasion can upregulate Bmspz4 expression in the integument. This study showed that Bmspz4 gene plays an important role in the innate immunity of integument of silkworm, Bombyx mori.

\section{Acknowledgments}

This work was supported by the National Natural Science Foundation of China (31272508).

\section{References}

[1] H. Jiang, A. Vilcinskas, M.R. Kanost, Immunity in lepidopteran insects, Advances in experimental medicine and biology, 708 (2010) 181-204.

[2] J.A. Hoffmann, The immune response of Drosophila, Nature, 426 (2003) 33-38. 
[3] D. Kang, G. Liu, A. Lundstrom, E. Gelius, H. Steiner, A peptidoglycan recognition protein in innate immunity conserved from insects to humans, Proc. Natl. Acad. Sci. U. S. A., 95 (1998) 10078-10082.

[4] J. Royet, J.M. Reichhart, J.A. Hoffmann, Sensing and signaling during infection in Drosophila, Current opinion in immunology, 17 (2005) 11-17.

[5] B. Lemaitre, J. Hoffmann, The host defense of Drosophila melanogaster, Annual review of immunology, 25 (2007) 697-743.

[6] A. Lundstrom, G. Liu, D. Kang, K. Berzins, H. Steiner, Trichoplusia ni gloverin, an inducible immune gene encoding an antibacterial insect protein, Insect biochemistry and molecular biology, 32 (2002) 795-801.

[7] M.P. Belvin, K.V. Anderson, A conserved signaling pathway: the Drosophila toll-dorsal pathway, Annual review of cell and developmental biology, 12 (1996) 393-416.

[8] M.S. Halfon, C. Hashimoto, H. Keshishian, The Drosophila toll gene functions zygotically and is necessary for proper motoneuron and muscle development, Developmental biology, 169 (1995) 151-167.

[9] R. Chasan, K.V. Anderson, The role of easter, an apparent serine protease, in organizing the dorsal-ventral pattern of the Drosophila embryo, Cell, 56 (1989) 391-400.

[10] A.N. Weber, S. Tauszig-Delamasure, J.A. Hoffmann, E. Lelievre, H. Gascan, K.P. Ray, M.A. Morse, J.L. Imler, N.J. Gay, Binding of the Drosophila cytokine Spatzle to Toll is direct and establishes signaling, Nature immunology, 4 (2003) 794-800.

[11] D. Morisato, K.V. Anderson, The spatzle gene encodes a component of the extracellular signaling pathway establishing the dorsal-ventral pattern of the Drosophila embryo, Cell, 76 (1994) 677-688.

[12] S. Mulinari, U. Hacker, C. Castillejo-Lopez, Expression and regulation of Spatzle-processing enzyme in Drosophila, FEBS letters, 580 (2006) 5406-5410.

[13] I.H. Jang, N. Chosa, S.H. Kim, H.J. Nam, B. Lemaitre, M. Ochiai, Z. Kambris, S. Brun, C. Hashimoto, M. Ashida, P.T. Brey, W.J. Lee, A Spatzle-processing enzyme required for toll signaling activation in Drosophila innate immunity, Developmental cell, 10 (2006) 45-55.

[14] F. Leulier, C. Parquet, S. Pili-Floury, J.H. Ryu, M. Caroff, W.J. Lee, D. Mengin-Lecreulx, B. Lemaitre, The Drosophila immune system detects bacteria through specific peptidoglycan recognition, Nature immunology, 4 (2003) 478-484.

[15] C.A. Janeway, Jr., Pillars article: approaching the asymptote? Evolution and revolution in immunology. Cold spring harb symp quant biol. 1989. 54: 1-13, Journal of immunology (Baltimore, Md.: 1950), 191 (2013) 4475-4487.

[16] V. Gobert, M. Gottar, A.A. Matskevich, S. Rutschmann, J. Royet, M. Belvin, J.A. Hoffmann, D. Ferrandon, Dual activation of the Drosophila toll pathway by two pattern recognition receptors, Science, 302 (2003) 2126-2130.

[17] Y.S. Kim, J.H. Ryu, S.J. Han, K.H. Choi, K.B. Nam, I.H. Jang, B. Lemaitre, P.T. Brey, W.J. Lee, Gram-negative bacteria-binding protein, a pattern recognition receptor for lipopolysaccharide and beta-1,3-glucan that mediates the signaling for the induction of innate immune genes in Drosophila melanogaster cells, The Journal of biological chemistry, 275 (2000) 32721-32727.

[18] T. Michel, J.M. Reichhart, J.A. Hoffmann, J. Royet, Drosophila Toll is activated by Gram-positive bacteria through a circulating peptidoglycan recognition protein, Nature, 414 (2001) 756-759.

[19] M.R. Kanost, H. Jiang, X.Q. Yu, Innate immune responses of a lepidopteran insect, Manduca sexta, Immunological reviews, 198 (2004) 97-105.

[20] L. Wang, P. Ligoxygakis, Pathogen recognition and signalling in the Drosophila innate immune response, Immunobiology, 211 (2006) 251-261.

[21] D. Morisato, K.V. Anderson, Signaling pathways that establish the dorsal-ventral pattern of the Drosophila embryo, Annual review of genetics, 29 (1995) 371-399.

[22] P. Ligoxygakis, N. Pelte, J.A. Hoffmann, J.M. Reichhart, Activation of Drosophila Toll during fungal infection by a blood serine protease, Science, 297 (2002) 114-116.

[23] B. Moussian, S. Roth, Dorsoventral axis formation in the Drosophila embryo--shaping and transducing a morphogen gradient, Curr. Biol., 15 (2005) R887-899.

[24] A. Hoffmann, A. Funkner, P. Neumann, S. Juhnke, M. Walther, A. Schierhorn, U. Weininger, J. Balbach, G. Reuter, M.T. Stubbs, Biophysical characterization of refolded Drosophila Spatzle, a cystine knot protein, reveals distinct properties of three isoforms, The Journal of biological chemistry, 283 (2008) 32598-32609.

[25] X. Hu, Y. Yagi, T. Tanji, S. Zhou, Y.T. Ip, Multimerization and interaction of Toll and Spatzle in Drosophila, Proc. Natl. Acad. Sci. U. S. A., 101 (2004) 9369-9374.

[26] T.C. Cheng, Y.L. Zhang, C. Liu, P.Z. Xu, Z.H. Gao, Q.Y. Xia, Z.H. Xiang, Identification and analysis of Toll-related genes in the domesticated silkworm, Bombyx mori, Developmental and comparative immunology, 32 (2008) 464-475.

[27] K. Taniai, T. Ishii, M. Sugiyama, A. Miyanoshita, M. Yamakawa, Nucleotide sequence of 5'-upstream region and expression of a silkworm gene encoding a new member of the attacin family, Biochemical and biophysical research communications, 220 (1996) 594-599.

[28] Y. Wang, T. Cheng, S. Rayaprolu, Z. Zou, Q. Xia, Z. Xiang, H. Jiang, Proteolytic activation of pro-spatzle is required for the induced transcription of antimicrobial peptide genes in lepidopteran insects, Developmental and comparative immunology, 31 (2007) 1002-1012.

[29] P. Tzou, S. Ohresser, D. Ferrandon, M. Capovilla, J.M. Reichhart, B. Lemaitre, J.A. Hoffmann, J.L. Imler, Tissue-specific inducible expression of antimicrobial peptide genes in Drosophila surface epithelia, Immunity, 13 (2000) 737-748.

[30] E.A. Levashina, E. Langley, C. Green, D. Gubb, M. Ashburner, J.A. Hoffmann, J.M. Reichhart, Constitutive activation of toll-mediated antifungal defense in serpin-deficient Drosophila, Science, 285 (1999) 1917-1919.

[31] M.M. Davis, Y. Engstrom, Immune response in the barrier epithelia: lessons from the fruit fly Drosophila melanogaster, J Innate Immun, 4 (2012) 273-283. 